in: Huchler, Norbert (Hg.) 2008: Ein Fach wird vermessen.

Positionen zur Zukunft der Disziplin Arbeits- und Industriesoziologie.

Berlin: edition sigma, S. 21-30

\title{
Von der Krise, dem Elend und dem Ende der Arbeits- und Industriesoziologie
}

Stefan Kühl

Die Klagen über eine „Krise der Industriesoziologie“ (vgl. Touraine 1972, S 25ff.), über das „Elend der Industriesoziologie“ (vgl. Herkommer 1972, S. 71) oder gar ein drohendes „Ende der Industriesoziologie“ (vgl. Brandt 1990, S. 272) sind fast so alt wie die Industrie- und Arbeitssoziologie als etablierte soziologische Teildisziplin. Ähnlich wie in anderen Bindestrichsoziologien - man denke nur an die Diskussionen in der Entwicklungssoziologie (vgl. Menzel 1992 , S. 23f.) - herrscht seit längerem ein munterer Alarmismus, in dessen Zuge regelmäßig das drohende Ende der Teildisziplin heraufbeschworen wird.

Das besondere Dilemma der Arbeits- und Industriesoziologie scheint darin zu liegen, dass sie mit dem Marxismus eine Theorie zur Verfügung hatte, die es ermöglichte, die drei Ebenen Gesellschaft, Betrieb und Person theoretisch zusammenzuklammern, dass aber andererseits die Verhaftung in einer Marxschen Theorietradition zu einer Immunisierung gegenüber Veränderungen ihres Gegenstandsbereiches führte (vgl. Brandt 1990, S. 267f.). Die immer komplexeren Befunde, die die Arbeits- und Industriesoziologie durch ihre eigenen Forschungen zutage förderte, konnten, so jedenfalls die These einiger Arbeits- und Industriesoziologen, in dem marxistischen Theorierahmen, von dem sich die Forschungen leiten ließen, nicht mehr sinnvoll interpretiert werden (vgl. Deutschmann 2002, S. 21).

Mit der zunehmenden Ausdifferenzierung der Gesellschaft in eigenständige gesellschaftliche Teilbereiche wurde die marxistische Leitdifferenz von Kapital und Arbeit immer fragwürdiger. Bei aller Wichtigkeit von Tarifauseinandersetzungen zwischen Arbeitgebern und Arbeitnehmern für interne Beziehungen in Organisationen schien sich diese Auseinandersetzung nicht als Ordnungsschema für die Gesamtgesellschaft zu eignen. Betriebe schienen deutlich mehr zu sein als der Ort, an dem die gesamtgesellschaftlich bestimmten Auseinandersetzun-

1 Bei diesem Artikel handelt es sich um einen Vortrag, den ich im Rahmen der von Hans Pongratz organisierten Reihe „Perspektiven der Industriesoziologie“ am ISF in München gehalten habe. Er beinhaltet konzeptionelle Überlegungen für ein Lehrbuch Arbeits- und Industriesziog Kolleginnen und Kollegen vom ISF in München sei herzlich für die anregende Diskussion der Thesen gedankt. Bernhard Gill, Sebastian Herkommer, Boris Holzer, André Kieserling, Manfred Moldaschl, Veronika Tacke, Georg Vobruba und Christof Wehrsig danke ich für Anmerkungen und Anregungen zu früheren Fassungen dieses Artikels. 
gen zwischen Kapital und Arbeit aufeinander prallten (vgl. Gergs et al. 2000, S. 189f.). Individuen ließen sich zunehmend schwerer in die durch die Produktionsverhältnisse geprägten Klassen einordnen, weil sie vieles stärker zu beeinflussen schien als ihr Status als Lohnarbeiter (vgl. auch Offe 1984, S. 16ff.).

Die Arbeits- und Industriesoziologie ist schon seit längerem in der Zwickmühle, „Marx in toto zu übernehmen“ und sich so des Vorwurfes eines „Ableitungsmarxismus" auszusetzen oder Karl Marx als Orientierungspunkt aufzugeben und damit die theoretische Klammer zu verlieren, aufgrund der die Arbeitsund Industriesoziologie immer den Anspruch vertreten konnte, mehr als eine simple Bindestrich-Soziologie zu sein (vgl. Bahrdt 1982, S. 14).

\section{Zwischen Enttheoretisierung und gesellschaftstheoretischem Anspruch: \\ Entwicklungsszenarien}

Wie die Arbeits- und Industriesoziologie sich weiterentwickeln wird, zeichnet sich lediglich in Konturen ab. Für eine wissenschaftliche Teildisziplin wäre es auch überraschend, wenn es eine einheitliche Antwort auf das oben genannte Dilemma gäbe. Nur Forschungsinstitute können als Organisationen über eine mehr oder minder funktionierende Hierarchie ihren Nachwuchsforschern Themen wie neue Produktionskonzepte, Paradoxien des Kapitalismus oder Autopoiesis in der Weltgesellschaft „diktieren“, wissenschaftlichen Disziplinen insgesamt fehlt die hierarchische Spitze, die Themen und Ansätze verordnen könnte.

Grob lassen sich innerhalb der Disziplin drei Entwicklungsrichtungen verzeichnen.

Die erste Entwicklungsrichtung lässt sich als „Enttheoretisierung“ der Arbeits- und Industriesoziologie bezeichnen. Angesichts der Probleme mit den marxistischen Zentralkategorien und eines zunehmend elaborierten Methodenapparates gibt es eine Tendenz, auf eine theoretische Einbettung zu verzichten und $z u$ den eher theoriereduzierten Herangehensweisen der Arbeits- und Industriesoziologie in der Nachkriegszeit zurückzukehren (vgl. Methodenüberblick bei Kühl/Strodtholz 2002). Gerade in den für die Arbeits- und Industriesoziologie wichtigen nicht-universitären Instituten scheint es in dem Spagat zwischen Akzeptanz durch die wissenschaftliche Gemeinschaft einerseits und der Verwendbarkeit außerhalb der Wissenschaft andererseits eine deutliche Verschiebung zu letzterer zu geben. Durch die Aufgabe eines klaren (auch nur betriebsoder organisations-)theoretischen Fokus und des Anspruches, mehr zu sein als eine ,unpraktische Wissenschaft", ähneln nicht wenige industriesoziologische Untersuchungen immer mehr den Gutachten von Unternehmensberatungen (vgl. Deutschmann 2002, S. 24ff.).
Die zweite Entwicklungsrichtung versucht, ihren Halt in Theorien mittlerer Reichweite zu finden. Weil Themen wie betriebliches Lernen, Projektarbeit, Vernetzung von Unternehmen oder Qualifikation von Management tendenziell nicht oder nur unter größten Schwierigkeiten mit Marxschen Kategorien gefasst werden können, wird das Heil in Theorien gesucht, die nicht mehr auf die Gesellschaft als Ganzes ausgerichtet sind, aber trotzdem für eine auf den Betrieb oder einen Teilaspekt der Wirtschaft gerichtete Fragestellung soziologischen Halt bieten (vgl. Deutschmann 2002, S. 40). Die Prominenz der Mikropolitik, der Steuerungstheorien oder der Governance-Ansätze in den arbeits- und industriesoziologischen Arbeiten der letzten Jahre ist nicht zuletzt diesen Bemühungen geschuldet. Die aus diesen Theorieperspektiven forcierten Forschungen waren zwar hochinnovativ, doch den Anspruch, Arbeits- und Industriesoziologie als umfassende Theorie voranzutreiben, konnten (und wollten) sie nicht einlösen. Eine an einer Vielfalt von Theorien mittlerer Reichweite orientierte Arbeitsund Industriesoziologie werde es, so die Beobachtung von Veronika Tacke, sicherlich nie an Arbeit fehlen, aber ihr kognitiver Kern löse sich in einem ,bunten Gemenge aus empirischer Organisations-, Wirtschafts-, Arbeits-, Technikund Politikforschung" auf (vgl. Tacke 2000, S. 124).

Die dritte Entwicklungsrichtung besteht in einer gesellschaftstheoretischen „Re-Fundierung“ der Arbeits- und Industriesoziologie. Von einer Gesellschaftstheorie kann man erst dann sprechen, wenn eine Theorie die Stabilität und den Wandel von Gesellschaft erklären und Makrokonflikte einordnen kann. Eine Gesellschaftstheorie muss über die Grundzüge einer Wirtschaftstheorie verfügen können und gleichzeitig in der Lage sein, das Verhältnis von Wirtschaft zu Politik, Recht, Erziehung, Wissenschaft, Religion und Massenmedien zu erklären. Sie muss die Funktionsweise von Organisationen, seien es nun Unternehmen, Schulen, Universitäten, Kirchen oder Fernsehanstalten, erklären können. Eine Gesellschaftstheorie darf sich nicht auf diese „Makroebene" und „Mesoebene" beschränken, sondern muss einen theoretisch geschulten Blick für so elementare Sozialformen wie das Einkaufen in Supermärkten, das Schmusen auf der Betriebsfeier oder die konfliktuösen Interaktionen in Klassenräumen entwickeln. Schließlich braucht eine Gesellschaftstheorie eine ausgearbeitete, Wissenssoziologie, mit der sie erklären kann, wie sie zu der Erkenntnis kommt, zu der sie kommt.

\section{Plädoyer für eine Rückkehr zur Gesellschaftstheorie in der Arbeits- und Industriesoziologie}

Die Stärke der marxistischen Theorie ist gewesen, dass sie schon in den Grundformulierungen von Karl Marx und Friedrich Engels einen Großteil der Ansprü- 
che an eine Gesellschaftstheorie bedienen konnte. Die marxistische Theorie besitzt mit der Idee des Klassenkampfes ein Konzept, mit dem Konflikte in der Weltgesellschaft, in den Nationalstaaten, in den Betrieben, Universitäten oder Fernsehanstalten und in Interaktionen zwischen Menschen erklärt werden konnten. Diese an Klassengegensätzen festgemachte Konfliktkonzeption dient in der marxistischen Theorie dazu, auch gesellschaftlichen Wandel zu beschreiben. Wie kaum eine andere soziologische Theorie verfügt der Marxismus über ein ausgefeiltes Verständnis kapitalistischer Wirtschaft und ist über die Unterscheidung von ökonomischer Basis und ideologischem Überbau in der Lage, das Verhältnis von Wirtschaft $\mathrm{zu}$ anderen gesellschaftlichen Teilbereichen zu bestimmen. Es ist das Verdienst der frühen Arbeits- und Industriesoziologie, dass sie die eher auf große gesellschaftliche Linien ausgerichtete marxistische Theorie auf die Ebene von Organisationsanalysen und teilweise auch auf die Ebene von konkreten Face-to-Face-Interaktionen (vgl. z.B. Burawoy 1979 oder auch Willis 1982) herunterbrach. Weiterhin verfügte der Marxismus mit seiner Überlegung, dass das Bewusstsein von Personen aus den Klassenlagen abgeleitet werden kann, über eine Konzeption, mit der Wissens- und Denkweisen rekonstruiert werden konnten. Es mag manchen Soziologen heutzutage zu brachial erscheinen, die Art und Weise, wie Leute denken und welches Wissen ihnen einleuchtet, aus der Klassenlage zu erklären. Es lässt sich aber nicht leugnen, dass der Marxismus mit diesem Gedanken über eine eigene komplette Wissenssoziologie verfügte (vgl. Kieserling 2003, S. 6ff.).

Die Prominenz des Marxismus als Gesellschaftstheorie wird daran erkennbar, dass sich alle soziologischen Klassiker - von Georg Simmel über Émile Durkheim bis hin zu Max Weber - an der Marxschen Theorie abgearbeitet haben. Nicht so sehr wissenschaftliche Kontroversen, sondern eher der Zusammenbruch des Staatssozialismus hat Karl Marx als Theoretiker aus dem allgemeinen Interesse verdrängt. Das ist zeitgeschichtlich verständlich, aber von der Sache her paradox (vgl. Haug 2002, S. 229). Vielleicht stimmt es, wenn Gert Schmidt (1990, S. 9) konstatiert, dass die an Karl Marx orientierten Soziologen seit geraumer Zeit an „Atemnot" litten und sich immer weniger empirische Evidenzen den klassentheoretisch nahe liegenden wissenschaftlichen Erklärungen fügten. Aber die Frage ist, ob hier Marx nicht als soziologischer Theoretiker etwas vorschnell beerdigt wird. Denn die Stärken der Marxschen Theorie liegen wohl weniger in einer marxistisch begründeten Betriebssoziologie oder einer arbeitssoziologischen Klassenbewusstseinsforschung, sondern eher in der Beschreibung der Expansionskraft einer kapitalistischen Wirtschaftsordnung.

Abgesehen von der Rational Choice Theorie, die mit ihrer ökonomischen Theoriebasis dem Marxismus viel ähnlicher ist, als man auf den ersten Blick vermuten mag, gibt es zurzeit wohl nur eine Gesellschaftstheorie, die ähnlich komplett ausgearbeitet ist wie der Marxismus: die Theorie funktionaler Diffe- renzierung. Die Theorie funktionaler Differenzierung wird heutzutage mit der Systemtheorie Niklas Luhmanns identifiziert, ihre Grundlagen finden sich jedoch bereits bei den am Ende des neunzehnten, Anfang des zwanzigsten Jahrhunderts wirkenden Soziologen Emile Durkheim und Max Weber (vgl. Schimank 2000, S. 27ff.). Durkheim hatte moderne Gesellschaften mit höheren Lebewesen verglichen, in denen verschiedene, arbeitsteilige Organe jeweils eine Funktion für das große Ganze erfüllen. In der modernen Gesellschaft bildete sich, so Durkheim, eine „organische Solidarität“ aus, weil die verschiedenen „Organe" gerade aufgrund ihrer Arbeitsteilung aufeinander angewiesen sind (vgl. Durkheim 1988, S. 236ff.). Auch Weber hatte bereits früh argumentiert, dass in der modernen Gesellschaft verschiedene „Wertsphären" wie die Wissenschaft, die Wirtschaft, das Recht, die Politik und die Kunst miteinander in Widerstreit geraten (Weber 1990, S. 536ff.).

Wo liegen die Stärken der Theorie funktionaler Differenzierung, besonders in der Luhmannschen Prägung? Im Gegensatz zu Theorien, die entweder einzelne Phänomene wie Organisationen (z.B. der Neo-Institutionalismus mit seinem Fokus auf den Diffusionsprozess von Organisationsmustern) oder das Verhältnis von Struktur und Handlung (z.B. den Akteur-Struktur-Bezug in der Strukturationstheorie) zu einer Gesellschaftstheorie erheben, hat die Theorie funktionaler Differenzierung den Vorteil, dass sie die Funktionsweise von Wirtschaft, Organisationen und Interaktionen in ihrer Spezifik beschreibt, ohne darüber auf den Anspruch, eine Gesellschaftstheorie zu sein, verzichten zu müssen. Die Theorie funktionaler Differenzierung bietet so die Chance, die Aspekte, für die sich die Arbeits- und Industriesoziologie interessiert, präziser in das Blickfeld zu bekommen, weil diese nicht von kausalen, doch relativ simplen Annahmen über die Auswirkung der Produktionsverhältnisse abgeleitet werden müssen. Erst wenn man von der Differenzierung der modernen Gesellschaft in weitgehend autonome gesellschaftliche Teilsysteme ausgeht, kann man die Rolle der Ökonomie in der Gesellschaft näher bestimmen. Erst wenn man das Eigenleben der Organisation betrachtet, erschließt sich die Frage nach der Funktion von Profit für Unternehmen. Erst wenn nicht die Klasse, sondern die Rolle als Nexus von Individuum und Gesellschaft begriffen wird, kann man das Zusammenspiel näher in Augenschein nehmen.

Es ist in der Selbstverständigung der Arbeits- und Industriesoziologie immer wieder darauf aufmerksam gemacht worden, dass der Einfluss dieser Disziplin von ihrer gesellschaftstheoretischen Fundierung abhängt (vgl. Herkommer 1972, S. 85; Lutz/Schmidt 1977, S. 219; Pries 1991, S. 19; Ortmann 1994, S. 105; Schumann 2003, S. 164ff.). Solange diese gesellschaftstheoretischen Anbindungen der Arbeits- und Industriesoziologie nicht deutlich herausgearbeitet werden und die Tendenz zu einer theoriegeminderten Darstellung der eigenen Disziplin fortgesetzt wird, wird sie über den eigenen Spezialistenkreis hinaus 
kaum wahrgenommen werden. Eine Arbeits- und Industriesoziologie, die sich auf die Pflege empirischer Steckenpferde, die genaue Beobachtung von Rationalisierungstrends oder die Verwaltung großer Forschungs- und Gestaltungsprogramme konzentriert, läuft Gefahr, dass die Erkenntnisse der Teildisziplin in der Soziologie insgesamt folgenlos bleiben (vgl. auch Hirsch-Kreinsen 2003, S. 6)

Welche (und ob überhaupt eine) der beiden hier dargestellten gesellschaftstheoretischen Perspektiven sich durchsetzen kann, hängt maßgeblich davon $a b$, ob es einer von beiden gelingt, die jeweils andere Perspektive zu integrieren (vgl. Schimank 1998). Das theoretische Schisma zwischen der marxistischen Theorie und der Theorie funktionaler Differenzierung führt im schlechtesten Fall $\mathrm{zu}$ einem gegenseitigen Anschweigen oder einer fortgesetzten pauschalen $\mathrm{Zu}$ rückweisung, im besten Fall zu produktiven Versuchen einer Integration der jeweils anderen Beobachterperspektive.

\section{Zum ,kritischen Potenzial“ der Arbeits- und Industriesoziologie}

Besonders groß scheint die Sorge zu sein, dass der Arbeits-, Betriebs- und Industriesoziologie das „kritische Potenzial“ verloren gehen könnte (vgl. zuletzt Schumann 2003, S. 157). Aber welche Form von Kritik ist gemeint, wenn vom „kritischen Potenzial“ der Arbeits- und Industriesoziologie die Rede ist? Geht es um eine Soziologie, die sich nicht von den bürgerlichen Selbstverständlichkeiten blenden lässt? Geht es um eine Soziologie, die die Proletarierin als "natürliche“ Koalitionspartnerin (an-)erkennt und mit ihr zusammen die kapitalistischen Produktionsverhältnisse revolutioniert oder wenigstens reformiert? Geht es um eine Soziologie, die sich in Fragen einer Humanisierung der Arbeitswelt nicht nur auf die Rolle des Begleitforschers beschränkt, sondern energisch Position für den Arbeiter bezieht?

Aus der Perspektive der Theorie funktionaler Differenzierung würde man argumentieren, dass die Soziologie als ausdifferenzierte und theoretisch abgesicherte Wissenschaft durchaus eine kritische Distanz zu ihrem Gegenstand hat. Es ist für eine ausdifferenzierte Wissenschaft charakteristisch, dass man sich primär für das Urteil seiner Fachkollegen interessiert und nicht besonders dafür, ob der Personalmanager, Gewerkschaftsfunktionär oder gar der Arbeiter am Fließband die eigenen Forschungen besonders gut und einleuchtend findet. Der Wissenschaftler schielt auf das Literaturverzeichnis des Aufsatzes seines Kollegen, um festzustellen, ob seine eigenen mehr oder minder innovativen Gedanken auch entsprechend gewürdigt wurden. Natürlich kann man sein Renommee auch aus der Prominenz in den Etagen der politischen Parteien, der Arbeitgeberverbände, der Gewerkschaften oder der Massenmedien ziehen, aber es fällt auf, dass in einer ausdifferenzierten Wissenschaft dieses Renommee nur schwer in wissen- schaftliches Renommee rückübersetzbar ist und Prominenz außerhalb der Wissenschaft eher als Anzeichen wissenschaftlicher Fragwürdigkeit gewertet wird.

Die Soziologie gewinnt als Wissenschaft der Gesellschaft ihre Stärke gerade dadurch, dass sie Beschreibungen anfertigen kann, die keine Rücksicht auf die Handlungslogiken von gesellschaftlichen Teilbereichen, auf die Interessen von Organisationen oder gar von Personen nehmen müssen. Ja, sie zieht ihre Existenzberechtigung als Sozialwissenschaft gerade daraus, dass sie die jeweils systemloyalen Selbstbeschreibungen mit für die beschriebenen Funktionssysteme, Organisationen oder Interaktionen ketzerisch wirkenden Fremdbeschreibungen kontrastiert (vgl. Kieserling 2000, S. $77 \mathrm{ff}$.). Die Soziologie kann beispielsweise - anders als die Theologie - Religion als „Opium des Volks“ bezeichnen (Marx 1956, S. 378) oder proklamieren, dass die Menschen, wenn sie Gott anbeten, eigentlich die Funktionsweise der Gesellschaft anbeten (Durkheim 1981). Sie kann - anders als die Betriebswirtschaftslehre - die Profitorientierung von Unternehmen als Mythos bezeichnen oder gar in der Profitorientierung den Anfang allen Endes sehen (vgl. Marx 1962). Gerade weil die Soziologie ihren Fokus auf die Gesamtgesellschaft richtet, braucht sie sich nicht wie andere Disziplinen (siehe z.B. Betriebswirtschaftslehre, Politologie) durch ein Mindestmaß an Loyalität gegenüber dem beschriebenen gesellschaftlichen Teilbereich auszuzeichnen (vgl. Kieserling 2000, S. 45ff.). Angesichts dieser Fähigkeit zu ketzerischen Fremdbeschreibungen ist die Forderung nach einer „kritischen Soziologie“" eigentlich ein „weißer Schimmel", ein Pleonasmus.

Der „Kritikbegriff“ der Arbeits- und Industriesoziologie ist aber lange Zeit enger gefasst gewesen als dieser aus der Ausdifferenzierung der Soziologie als Sozialwissenschaft abgeleitete Kritikbegriff. Es ging um Kritik an den ,,herrschenden Verhältnissen" bei gleichzeitiger (natürlich auch kritischer) Loyalität gegenüber ,gesellschaftsverändernden“" Gruppen der Gesellschaft. Deutlich schimmerte die berühmte elfte Marxsche These über Feuerbach durch, dass es nicht darauf ankomme, Gesellschaft zu interpretieren, sondern darauf, sie zu verändern (vgl. Marx 1958, S. 535). Da der Kapital-Arbeit-Gegensatz das zentrale Differenzierungsschema dafür war, wem gegenüber man kritisch zu sein hatte, war das Problem der Arbeits- und Industriesoziologie eine abgeschwächte Distanz zu den Vertretern der Arbeitnehmerschaft. Diese wurde teilweise unter dem Label der „Arbeitnehmerorientierung“ und der Besetzung von soziologischen Universitätsstellen mit Personen mit einer Doppelqualifikation als Facharbeiter und Wissenschaftler in den Boomphasen Anfang der siebziger Jahre offen propagiert (vgl. schon die frühe Beobachtung von Hoefnagels 1966, S. $48 \mathrm{ff}$.).

Gegen diesen Entwicklungspfad hin zu einer affirmativen Reflexionstheorie der Gewerkschaften ist erst einmal nichts einzuwenden, hält sich das Management mit der dominierenden Betriebswirtschaftslehre ja ebenfalls eine eigene Reflexionstheorie, die sich aber aufgrund ihrer Praxisorientierung der Kritik der 
fehlenden wissenschaftlichen Distanz aussetzen muss. Aber genauso wie sich die Betriebswirtschaftslehre fragen lassen muss, ob sie durch ihren affirmativen Zugang die Unternehmen wirklich über sich selbst aufklärt, muss sich eine arbeitnehmerorientierte Arbeits- und Industriesoziologie fragen lassen, ob sie den Gewerkschaften als ihren „,natürlichen“ Ansprechpartnern wirklich nutzt, wenn sie mit der Gewerkschaftsprogrammatik vereinbare Beschreibungen wirtschaftlicher Prozesse anfertigt. Genauso wie in Teilen des Unternehmensmanagements der Trend zu beobachten ist, unter dem Label „Quäl mich“ mehr als „, sozialverträgliche Beschreibungen" von den Organisationswissenschaften einzufordern, stellt sich auch für die Gewerkschaften die Frage, ob es ihnen nicht mehr nützen würde, wenn die Arbeits- und Industriesoziologie ketzerische (weil distanzierte) Beschreibungen nicht nur über die kapitalistischen Unternehmen, sondern auch über die Gewerkschaften selbst anfertigte.

Diese Frage kann man getrost den Unternehmen und Gewerkschaften als Nachfragern arbeits-, betriebs- und industriesoziologischen Wissens überlassen. Für die Soziologie als Sozialwissenschaft ist die Begrenzung eines Blickwinkels problematisch. Der Kapital-Arbeit-Gegensatz als Leitdifferenz versetzt in die Lage, hierarchisch strukturierte Herrschaftsverhältnisse in Betrieben zu beschreiben, verkennt aber, dass die Gewerkschaften in ihrem professionalisierten Kern ähnliche hierarchische Prinzipien ausbilden. Man kommt über den KapitalArbeit-Gegensatz an die Entfremdungsphänomene in taylorisierten oder auch nicht taylorisierten Betrieben heran, verbaut sich aber tendenziell die Einsicht, dass auch in Interessensorganisationen in dem Moment, in dem sie Mitgliede über Lohnarbeit finanzieren, vergleichbare Entfremdungsphänomene auftreten können.

Der Charme einer soziologischen Absicherung im Marxismus oder in der Theorie funktionaler Differenzierung liegt darin, dass eine so theoretisch fundierte Soziologie erst einmal eine im besten Sinne ,unpraktische Wissenschaft" (Bergmann 1982) ist. Man kann geradezu die Stärke der Arbeits- und Industriesoziologie daran messen, als wie ketzerisch ihre Beschreibungen im beschriebenen Feld angesehen werden. Es ist ein Qualitätsmerkmal einer theoretisch abgesicherten Arbeits- und Industriesoziologie, dass ihre Erkenntnisse nicht einfach in die Praxis eines Unternehmens, einer Gewerkschaft oder auch einer sozialen Bewegung übertragbar sind, sondern dort als irritierende Aufklärung über sich selbst (und nicht nur über ihre Umwelt) empfunden werden. Der wissenschaftliche Reiz der Theorie funktionaler Differenzierung ist, dass sie anders als der Marxismus für Unternehmen und Gewerkschaften, für Kapital und Arbeit, für Wirtschaft und Politik in gleicher Weise „unpraktisch" und „ketzerisch" ist. Welche bessere Basis für eine wissenschaftliche Kritik könnte es geben?

Pahrdt, H P (1982): Die Industriesoziologie - eine ,spezielle Soziologie"'? In: Schmidt, G Braczyk, H.J. Knesebeck, J. v. d. (Hg): Materialien zur Industriesoziologie. Opladen, Braczyk,
S. $11-15$

Bergmann, J. (1982): Industriesoziologie - eine unpraktische Wissenschaft? In: Beck, U. $(\mathrm{Hg}$.$) : Soziologie und Praxis. Soziale Welt, Sonderband I, S. 397-416$

Brandt, G. (1990): Arbeit, Technik und gesellschaftliche Entwicklung. Transformationsprozesse des modernen Kapitalismus. Frankfurt/M.

Burawoy, M. (1979): Manufacturing Consent. Chicago, London

Deutschmann, Chr. (2002): Postindustrielle Industriesoziologie. Theoretische Grundlagen, Arbeitsverhältnisse und soziale Identitäten. Weinheim, München

Durkheim, E. (1981): Die elementaren Formen des religiösen Lebens. Frankfurt/M

Durkheim, E. (1988): Über soziale Arbeitsteilung. Studie über die Organisation höherer $\mathrm{Ge}-$ sellschaften. Frankfurt/M.

Gergs, H.; Pohlmann, M.; Schmidt, R. (2000): Organisationstheorie, ihre gesellschaftliche Relevanz und "gesellschaftstheoretische Herausforderung". In: Münch, R.; Jauß, C.; Stark, C. (Hg.): Soziologie 2000. Soziologische Revue, Sonderheft 5, S. 183-195

Haug, W. F. (2002): Karl Marx - der Kritiker des Kapitalismus. In: Das Argument Nr. 245, Jg. 44, H. 2, S. $229-240$

Herkommer S (1972): Vom Elend der Industriesoziologie. In: Sozialistische Politik, Jg. 16 H. 4, S. $71-88$

Hirsch-Kreinsen, H. (2003): Renaissance der Industriesoziologie? In: Soziologie, Jg. 32, H. 1, S. $5-20$

Hoefnagels, H. (1966): Soziologie des Sozialen. Einführung in das soziologische Denken. Essen Kieserling, A. (2000): Die Soziologie der Selbstbeschreibung. In: de Berg, H.; Schmidt, J. (Hg.): Rezeption und Reflektion. Frankfurt/M., S. 38-92

Kieserling, A. (2003): Schichtung und Klassenbildung. Mainz (unveröffentlichtes Manuskript)

Kühl, St. (2004): Arbeits- und Industriesoziologie. Bielefeld

Kühl, St.: Strodtholz P. (Hg.) (2002): Methoden der Organisationsforschung. Ein Handbuch. Reinbek

Lutz, B.; Schmidt, G. (1977): Industriesoziologie. In: König, R. (Hg.): Handbuch der empirischen Sozialforschung. München, S. 101-262

Marx, K. (1956): Zur Kritik der Hegelschen Rechtsphilosophie. Einleitung. In: Marx-EngelsWerke, Band 1. Berlin, S. 378-391

Marx K (1958): Thesen über Feuerbach. In: Marx-Engels-Werke, Band 3. Berlin, S. 533-535 Marx, K. (19. (1992): Das Ende der Dritten Welt und das Scheitern der großen Theorien. FrankMenzel, U. (1992)

Offe, C. (1984): Arbeit als soziologische Schlüsselkategorie? In: Offe, C. (Hg.), Arbeitsgesellschaft. Strukturprobleme und Zukunftsperspektiven. Frankfurt/M., New York, S. 13-43

Ortmann, G. (1994): Dark Stars - Institutionelles Vergessen in der Industriesoziologe. In: Beckenbach, N.; van Treek, W. (Hg.): Umbrüche gesellschaftlicher Arbeit. Soziale Welt, Sonderband 9, S. 85-118 